\title{
A Validated Method for Quantification of Fatty Acids Incorporated in Human Plasma Phospholipids by Gas Chromatography-Triple Quadrupole Mass Spectrometry
}

\author{
Hans-F. Schött, ${ }^{*}$, Maurice C. J. M. Konings, ${ }^{\S}$ Vera B. Schrauwen-Hinderling, Ronald P. Mensink,
} and Jogchum Plat*

Cite This: ACS Omega 2021, 6, 1129-1137

Read Online

ABSTRACT: Fatty acids (FA) are important mediators of health maintenance and disease risk. Optimal quantification assays of FA in high and low abundance as well the identification of ${ }^{13} \mathrm{C}$-labeled tracers to monitor FA metabolism are of major interest. The article on hand reports about the development and validation of a gas chromatography (GC) -triple quadrupole mass selective detection (GC-TQMS) method for absolute quantification of FA in human plasma phospholipids (hpPL). The quantification of the calibration solution by GC-flame ionization detection (GC-FID), with the introduction of a correction factor, allows the direct comparison of individual FA concentrations in hpPL by GC-TQMS. Specificity, sensitivity, and reproducibility are achieved by optimized chromatographic separation and employment of GC-TQMS. The

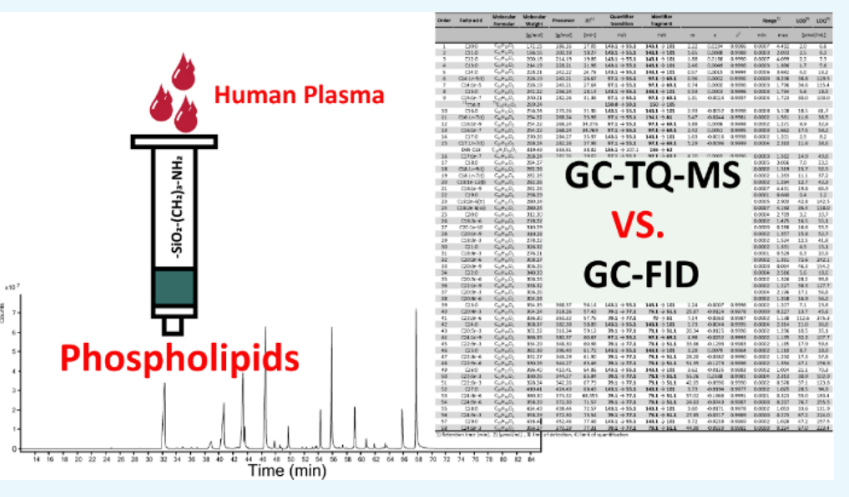
inter-method comparison between GC-FID and GC-TQMS concentrations revealed good comparability for 27 FA. A full validation has been performed with linearity over 4 magnitudes, a limit of detection of $0.18-38.3$ fmol on column, a recovery of $83.6-109.6 \%$, and intraday and interday precision data meeting the criteria of EMA and FDA guidelines. The method includes the absolute quantification of 58 positional and geometrical (cis/trans) isomeric FA in hpPL in the concentration range of $1-3000 \mathrm{nmol} / \mathrm{mL}$, covering also low abundant positional cis/trans isomers. Results obtained from both methods are highly comparable, and selectivity and sensitivity are improved by using GC-TQMS. Additionally, we show here that calculation of ${ }^{13} \mathrm{C}-$ labeled $\mathrm{C} 16: 0$ tracer/tracee ratios in hpPL in human isotope enrichment studies is possible.

\section{INTRODUCTION}

Fatty acids (FA) have always been at the center of research on cardiovascular diseases. Human diets contain a wide variety of FA, which enter the circulation after intestinal absorption, and are incorporated in tissues, mostly as membrane constituents amongst others, the phospholipids (PL). FA possess a variety of biological activities such as regulation of membrane structure and function; regulation of intracellular signaling pathways, transcription factor activity, and gene expression; and regulation of the production of bioactive lipid mediators. ${ }^{1}$ For example, poly-unsaturated FA play an important role in the life and death of cardiac cells and have a direct positive impact on the risk prevention of cardiovascular diseases, i.e., cardiac infarction. ${ }^{2-4}$ Moreover, poly-unsaturated $\omega-3$ and $\omega-6$ FA are also essential precursors for lipid signaling molecules, i.e., eicosanoids and endocannabinoids. ${ }^{5-7}$ Another example are the trans-FA, which impair cholesterol metabolism, reveal proinflammatory effects on endothelial cells, and are considered to increase insulin resistance and obesity. ${ }^{8-11}$ Observational studies also support the hypothesis of an interplay of trans-FA and coronary diseases. ${ }^{12}$ Given these functions, FA are important mediators of health maintenance and disease risk. Considering their fundamental importance for human health, optimal fatty acid identification and reliable quantification assays are of major interest as well as for the exploration of metabolic fluxes. Being able to quantify and compare concentrations of individual FA, preferably not only in human plasma phospholipids (hpPL) but also in different matrices will provide deeper insight in the role of fatty acid concentrations in various (patho)physiological processes. Nowadays, in most laboratory settings, ${ }^{13} \mathrm{C}$-labeled FA tracers in human absorption studies are commonly analyzed by applying two separate sequential assays like gas chromatographic assays and isotope-ratio mass spectrometry. It would be of great advantage if ${ }^{13} \mathrm{C}$-labeled FA and total FA in hpPL could

Received: August 22, 2020

Accepted: November 25, 2020

Published: January 4, 2021 
Table 1. Fifty-Eight Fatty Acids Included in GC-TQMS Assay for Fatty Acids Incorporated in Human Plasma Phospholipids ${ }^{a}$

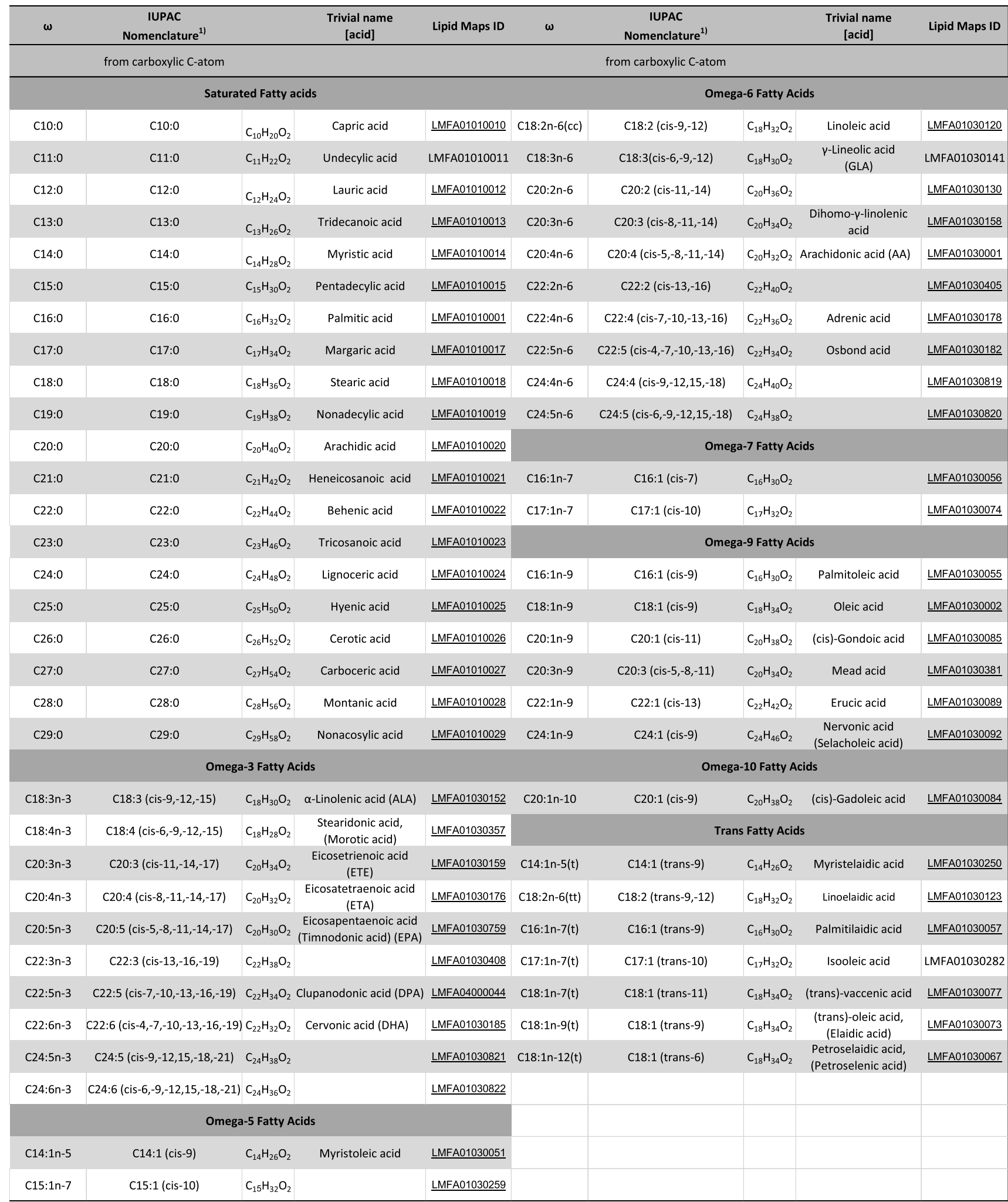

${ }^{a}$ Listed are the IUPAC nomenclature, sum formula, trivial names, and lipid map IDs.

be quantified in the same analytical assay to calculate tracer/ tracee ratios in human absorption studies. We describe here the analytic protocol for highly sensitive, specific, and robust absolute quantification of a large set of cis/trans positional isomeric FA in hpPL determined by gas chromatography-triple quadrupole mass selective detection (GC-TQMS) validated

under the consideration of EMA and FDA guidelines. 


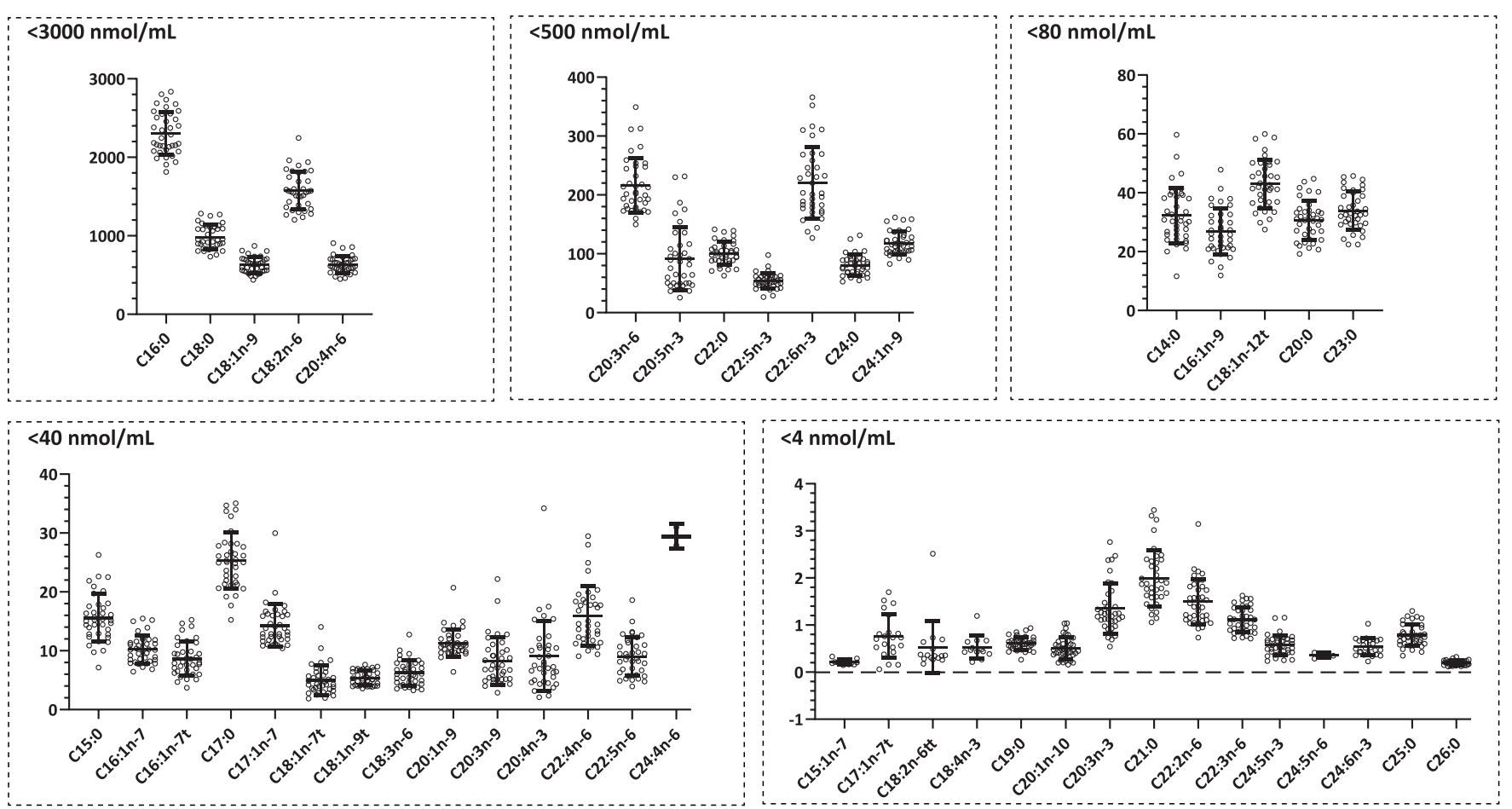

Figure 1. Concentrations (in $\mathrm{nmol} / \mathrm{mL}$ ) of 49 saturated and unsaturated cis/trans isomeric fatty acids in the range from C14 to C26 chain length. Boxplot parameters show the mean and the standard deviation of the fatty acid concentrations quantified in human phospholipids in 37 samples obtained from healthy volunteers. Phospholipids derived from human plasma were enriched by solid phase extraction on an amino propyl column. Fatty acids in enriched human phospholipid fraction were derivatized to fatty acid methyl ethers (FAMEs) prior to chromatographic separation on an Agilent 7890A gas chromatograph equipped with a CP-Sil 88 column ( $100 \mathrm{~m}$ in length, $0.25 \mathrm{~mm}$ in diameter, and $<0.192 \mu \mathrm{m}$ film thickness, Agilent J\&W). Fatty acid methyl esters were detected in positive multiple reaction monitoring mode (MRM) by quantifier and identifier transitions for saturated (143.1 $\rightarrow 55.1$ $\mathrm{m} / z ; 143 \rightarrow 101.1 \mathrm{~m} / z)$, mono-unsaturated $(97.1 \rightarrow 55.1 \mathrm{~m} / z ; 97.1 \rightarrow 69.1 \mathrm{~m} / z)$, and poly unsaturated $(79.1 \rightarrow 77.1 \mathrm{~m} / z ; 79.1 \rightarrow 51.1 \mathrm{~m} / z)$ fatty acid methyl esters. The total runtime was $85 \mathrm{~min}$, and FAMEs were quantified against an external calibration curve on an Agilent 7000 TQ-MS. As internal standard, a $\mathrm{D}_{35}$-C18:0 FAME was used.

\section{RESULTS AND DISCUSSION}

Here, we report the first GC-TQMS method for the absolute quantification of a set of $58 \mathrm{cis} /$ trans and geometrical isomeric FA. We describe here in detail the following: (1) methodological aspects of fatty acid determination and sample preparation, (2) the reproducible purification of phospholipids from human serum, (3) the preparation of external calibration solution for the absolute quantification of major and low abundant FA in hpPL, (4) the validation parameters under the consideration of international guidelines, (5) and finally the comparison of percentage fatty acid patterns obtained by gas chromatographyflame ionization detection (GC-FID) and GC-TQMS. Table 1 lists the IUPAC names, molecular formulas, trivial names, and lipid map IDs of the 58 included fatty acids.

Method Development. Fatty acid methyl esters (FAMEs) undergo a strong fragmentation by electron impact (EI) ionization at $70 \mathrm{eV}$ and from numerous fragments. Identifier and quantifier transitions were evaluated for sufficient abundancies, suitable for trace analysis. Intact precursor ions show relatively low abundance and are therefore not suitable for trace analysis of low abundant FA. Mass fragments for identification and quantification were selected, which show high abundance and the most specificity for the individual type of FA. Saturated FAMEs possess an abundant fragment at 143.1 $m / z$. Unsaturated long chain FA underwent stronger fragmentation than unsaturated short chain FA and saturated FA. Saturated, mono-unsaturated, and poly-unsaturated FAMEs were quantified and identified by two transitions: for quantifying by the precursor $\rightarrow$ quantifier transition and for purity/ identification by the precursor $\rightarrow$ identifier transition. Saturated FA were quantified with the transitions $143.1 \rightarrow 55.1 \mathrm{~m} / z$ and $143.1 \rightarrow 101.1 \mathrm{~m} / z$, mono-unsaturated FA were quantified by $97.1 \rightarrow 55.1 \mathrm{~m} / z$ and $97.1 \rightarrow 69.1 \mathrm{~m} / z$; poly-unsaturated by 79.1 $\rightarrow 77.1 \mathrm{~m} / z$ and $79.1 \rightarrow 51.1 \mathrm{~m} / z$, respectively. An increase in specificity and sensitivity might be realized by using chemical ionization as previously reported by $\mathrm{Xu}$ et al. and Kish-Trier et al.. ${ }^{13,14}$ However, Kish-Trier et al. did not report improvement in limits of detection (LOD) and limits of quantification (LOQ) compared to the here reported method. Details are listed in Supplemental Table S1.

Preparation of Internal Standard for Absolute Quantification of FAMEs. $\mathrm{D}_{70}$-C18:0 phosphatidylcholine (PC) was used as an internal standard (IS) for FA quantification within the hpPL. This PC consists of two deuterated $\mathrm{D}_{35}-\mathrm{C} 18: 0$ FA. First efforts were made to use the deuterated PL and the deuterated $\mathrm{D}_{35}$-C18:0 FAME for external calibration, from separately prepared batches. Evaluation of the deuteration pattern of the two labeled FA revealed significant differences. For a comparison of the deuteration patterns of available free FA and the deuterated phospholipid, see Supplemental Figure S3. Differences in the deuteration pattern within the $\mathrm{D}_{70}-\mathrm{C} 18: 0 \mathrm{PC}$ in the hpPL sample and the $\mathrm{D}_{35}$-C18:0 FAME would result in significant differences in the signal intensity. This circumstance would consequently impair proper absolute concentrations of FAMEs and introduce a systematic bias. Therefore, it is not recommended to use deuterated $\mathrm{FA}$ obtained from different 

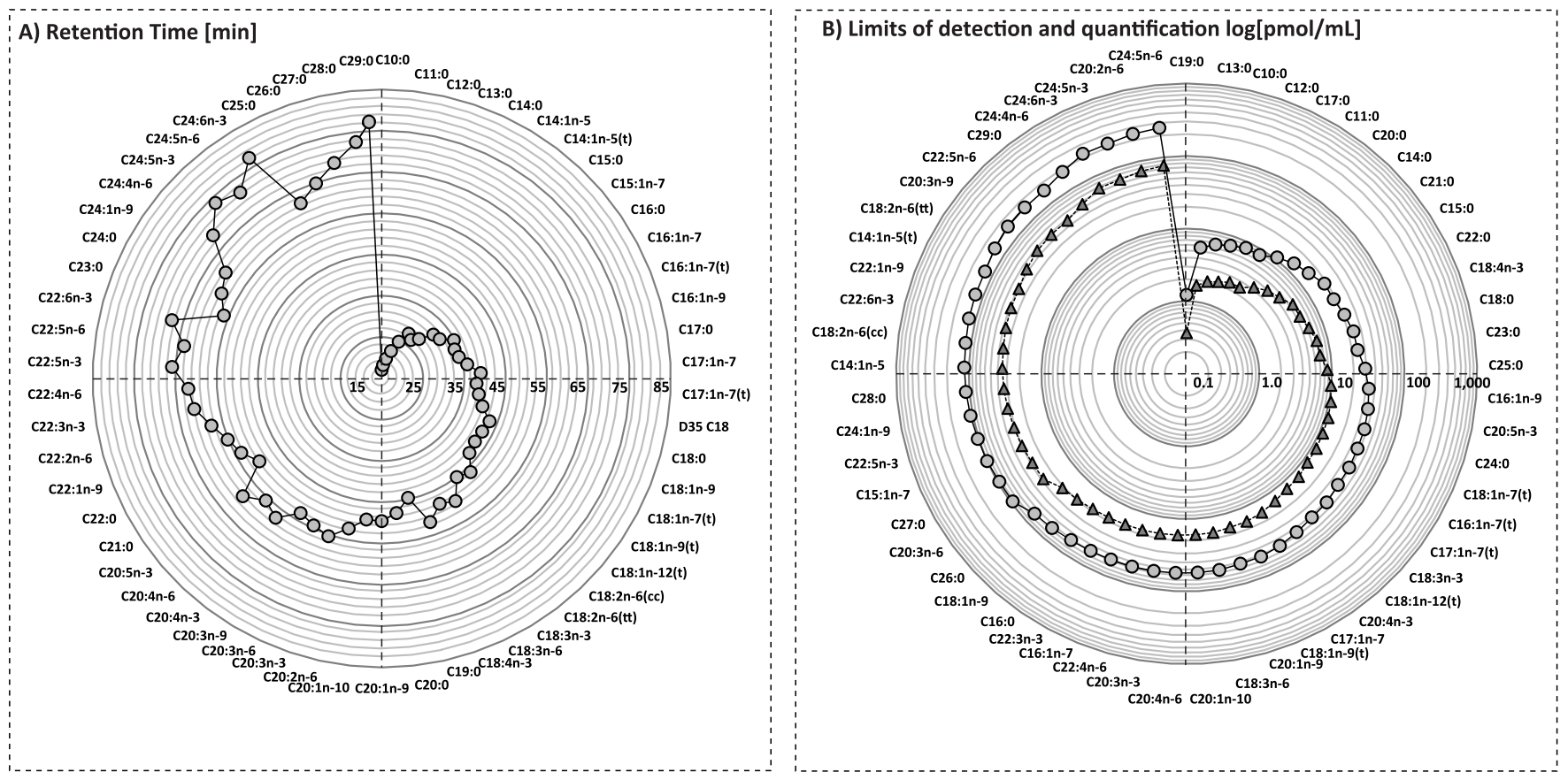

Figure 2. Retention time and limits of detection and quantification. (A) Retention time of included fatty acids ordered by chain length and saturation. (B) Limit of detection and quantification ordered by decreasing sensitivity.

batches or companies as the IS. To avoid the introduction of a systemic error, we synthesized the IS for the external calibration from the available $\mathrm{D}_{70}-\mathrm{C} 18: 0 \mathrm{PC}$. Please find the protocol for IS synthesis and the deuteration pattern of different FA in Supplemental Section 1. The obtained $\mathrm{D}_{35^{-}} \mathrm{C} 18: 0$ methyl ester was quantified by GC-FID against C19:0, and corresponding molarities of $\mathrm{D}_{35}-\mathrm{C} 18: 0$ and $\mathrm{D}_{70}-\mathrm{C} 18: 0 \mathrm{PC}$ were used in the serum samples and the external calibrations, respectively. The use of deuterated FA also allows the quantification of odd numbered or branched FA, i.e., C19:0 and C17:0. These fatty acids are often used as an IS in GC due to their low physiological concentrations in human plasma. The FA concentrations found in the volunteer cohort shown in Figure 1 are in good accordance to concentrations described by Khaw et al. ${ }^{15}$ They refer in the EPIC-Norfolk Prospective Study, that includes 10,000 cases, an average hpPL concentration of $4.768 \mathrm{mmol} / \mathrm{L}$. This is in good accordance to the average $7.416 \mathrm{mmol} / \mathrm{L}$ concentration in the study on hand. ${ }^{15}$ Khaw et al. used a GCMS-based assay with deuterated C16:0 as the IS. Regrettably, they do not describe the analytical method in detail nor indicate specifications of the deuterium labeled pattern of the used IS.

Preparation of External Calibration Solutions for Absolute Quantification of FAMEs. Customized external calibration solutions and Supelco 37 FAME mix, used for absolute quantification by GC-TQMS, were quantified by GCFID against C19:0 FAME. This additional step was needed to quantify the FAME concentrations in the custom-prepared calibration solutions. To normalize concentrations in these solutions with the same analytical method, it was necessary to introduce a correction factor (Rf), which considers differences in acyl chain length and the number of double bonds. de Saint Laumer et al. reported the prediction of response factors for GCFID from the individual combustion enthalpies. ${ }^{16}$ This calculation can be simplified since here structurally similar chemical compounds are compared. Detailed equations for calculating the correction factor are shown within Supplemental
Equation $\mathrm{S} 1$. We determined a good conformity $(\Delta-2.8 \%$ to $+9.8 \%)$ of $\mathrm{Rf}$ corrected concentrations and the reported concentrations of the Supelco 37 FAME mix. This commercially available solution is quantified by GC-FID on an SP 2560 (100 $\mathrm{m}, 0.25 \mathrm{~mm}, 0.2 \mu \mathrm{m}$ ) column operating with a helium flow at 30 $\mathrm{mL} / \mathrm{min}$, injected with a split ratio of $1: 100$, and pentadecane used as the IS. This good correlation of concentrations underlines the performance of our quantification method for the standards by GC-FID. The normalization of fatty acid concentrations allows also the direct comparison of individual FA concentrations within the sample. This will help for a detailed analysis of metabolic fluxes. The fatty acid quantification with an externally calibration curve and a deuterated IS allows one to report absolute molar concentrations of individual FA. This improvement enables the detailed investigations of metabolic changes within desaturation and prolongation of fatty acids.

Substantial Loss of FAMEs Shorter than C14:0 during Evaporation Was Observed. Therefore, a reliable absolute quantification of these medium-length FA derivatized to FAMEs must be carefully evaluated. Sample preparation that contains solvent evaporation steps after derivatization must be avoided. Considering this fact, further evaporation steps after the methylation have been avoided within the here described protocol. The FA C10:0, C11:0, and C13:0 were not detectable in the study samples nor in pooled plasma samples used for method validation. The loss of short-chain FA during sample evaporation is reported in detail in Supplemental Figure S4.

Method Validation. We evaluated the performance of the GC-TQMS method by an entire validation process according to the guidance for bioanalytic methods defined by the U.S. Food and Drug Administration and the European Medicines Agency. ${ }^{17,18}$ Validation parameters, namely, retention time, dynamic range, LOD and LOQ intra- and interday precision, volunteer concentrations, equations of calibration curve, correction factor Rf, molecular weight, and FAME precursor 


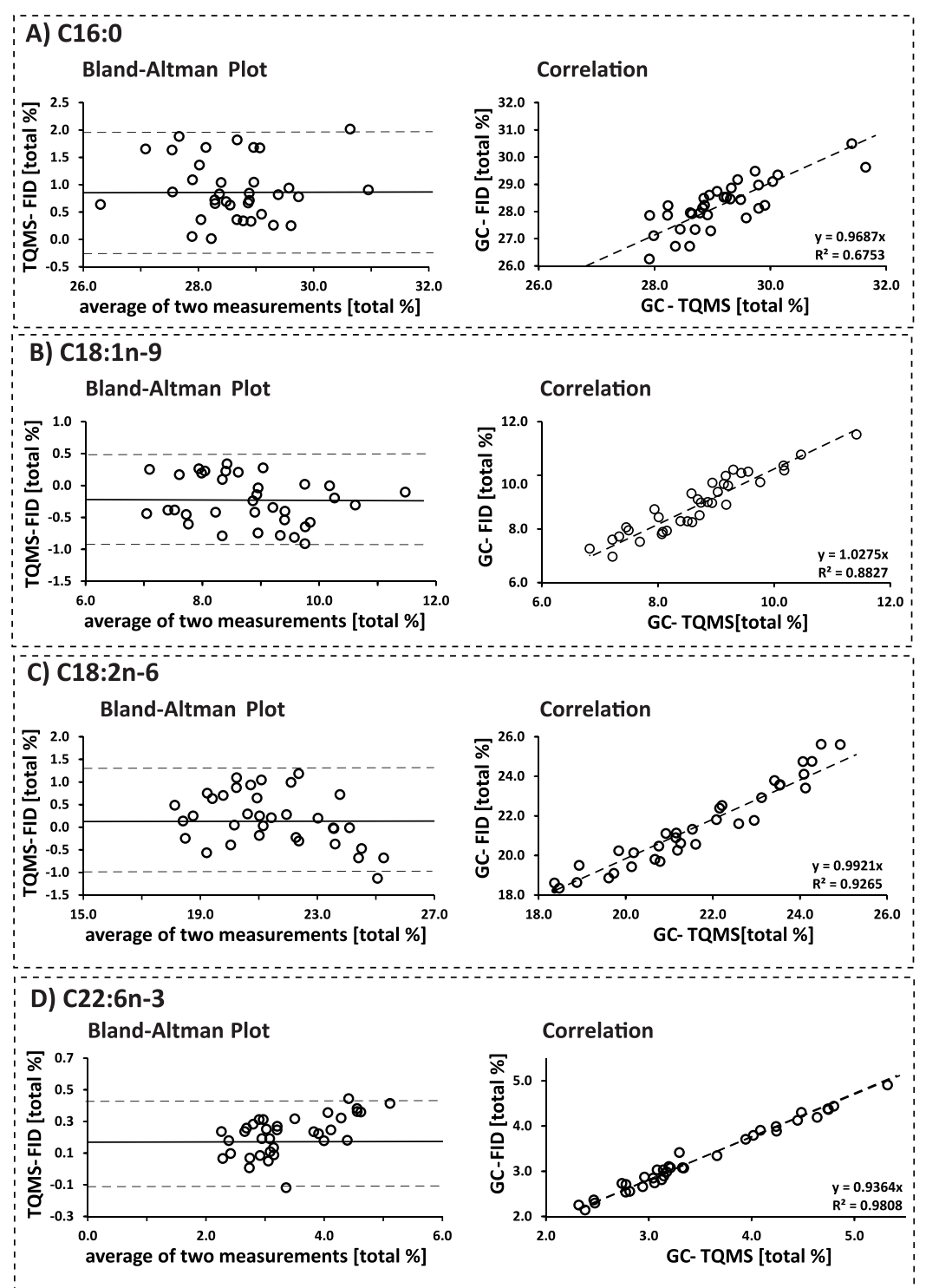

Figure 3. Bland-Altman plots and correlations for the most abundant saturated and $\omega-3, \omega-6$, and $\omega-9$ fatty acids in (A) to (D), respectively.

mass are shown in Figure 2 and listed in Supplemental Tables S1 and S2.

Selectivity. Specific retention times, individual transitions, and the ratios of identifier to quantifier areas allow an explicit identification of the analyzed FAMEs. The GC method was designed for baseline separation of all analytes (see Supplemental Figure S5). FAMEs that could not be separated by gas chromatography are identifiable by specific transitions. Using a column with a length of $100 \mathrm{~m}$, it was possible to separate positional isomeric fatty acids $\mathrm{C} 18: 1 \mathrm{n}-9(\mathrm{t}), \mathrm{C} 18: 1 \mathrm{n}-7(\mathrm{t})$, C18:1n-12(t), and C18:1n-9(c) from each other. As shown in Supplemental Figure S4. The retention time is shown in Figure 2A.

Linearity. All external calibration lines revealed a linearity within the quantification range greater than $R=0.99$, except for C18:1n-12(t) (with $R=0.8533$ ). A linear response was found in a dynamic range of 4 orders of magnitude from 0.04 to 5198 $\mathrm{nmol} / \mathrm{mL}$.

Limits of Quantification and Detection. The GC-TQMS method revealed good LOD and LOQ values with 0.4-112.6 and $1.2-375.3 \mathrm{pmol} / \mathrm{mL}$, respectively. The here reported LOD and LOQvalues are comparable to those described by Ecker et al., which are obtained from a single quadrupole mass spectrometer (MS). ${ }^{19}$ Moreover, the LOD and LOQ increased with FA chain length and degree of unsaturation and varied between 0.18 and 38.3 fmol (see Figure 2B). Saturated FA revealed the lowest LOD and LOQvalues due to the abundance of the main fragment $143.1 \mathrm{~m} / z$ (see Figure $2 \mathrm{~B}$ ). The equations for calculation of the LOD and LOQ are shown in Supplemental Equations S2 and S3.

Recovery. The recovery was assessed by spiking a pool serum with the commercially available Supelco phospholipid mixture in three different concentrations. Good recovery values of the 15 saturated and unsaturated FA between 83.6 and $109.6 \%$ were observed. This result indicates a robustness and reproducibility of sample preparation and fatty acid determination. The equation for calculation of the recovery is shown in Supplemental Equation S4.

Precision. The intraday and interday precision for FA in the pool serum were evaluated in the range of $0.9-15.7 \%$ (excluding C19:0) and 2.5-19.9\%, respectively. A precision value for six fatty acids absent in the pool serum could not be referred. 
Carry Over. After injection of the highest calibration point, no significant amounts of FA (below LOD) were discovered in the subsequent isooctane injection. Therefore, it can be assumed that no carry over of FA from previous runs will occur.

Due to the highly reproducible separation of FAMEs by gas chromatography and the additionally specific identification by qualifier and quantifier molecule fragments as well as the ratio of both fragments, the method disposes a high degree of selectivity and reveals therefore an advantage to comparable GC-FID or GC-MS assays. Our data proves the robust performance and the suitability of the method for absolute quantification of FA patterns in hpPL.

Method Comparison. Current well-established GC-FID and GC-MS methods have proven to be highly suitable for FA analysis by derivatization to FAMEs. However, these methods possess some limitations and restrictions. ${ }^{19-24}$ GC-FID assays reveal highly robust and quantitative FA analysis over a broad concentration range. FID enables the direct comparison of areas of the analyte and IS. By combusting organic compounds in a hydrogen flame, carbon atoms are thermally ionized and detected. Peak areas of individual analytes can be compared to the known concentration of an added IS. However, GC-FID shows limited sensitivity for FA in the lower concentration range. Also, the signal is relatively unspecific since flame ionization detectors respond on all hydrocarbons. ${ }^{25}$ To overcome this issue, the high separation performance of the GC has been coupled to MS. TQMS disposes a substantially increased sensitivity and specificity caused by the analysis of analyte-specific mass fragments. Due to the $\mathrm{MS}^{2}$ fragmentation, TQMS gives more information about the structure of an isolated mass fragment. Additionally, TQMS also possesses a lower background noise in $\mathrm{MS}^{2}$ mode. Both are reasons for the higher specificity and sensitivity of TQMS compared to the single quadrupole MS. In contrary to GC-FID assays, the areas obtained from analyte-specific mass fragments are not directly comparable to that from an IS. This circumstance is caused by several effects as ionization efficiency and fragmentation, which are specific for each analyte and result in a different response. Therefore, absolute concentrations of FA must be determined by external calibration solutions that are often custom prepared by the individual laboratory.

Fatty acid patterns are often referred as the molar percentage of a set of predefined FA. Thus, this reported pattern can be different between research groups. The comparability of the FA pattern (on a percentage basis) analyzed by the newly developed GC-TQMS method as well as by an established GC-FID method in our laboratory was evaluated. This experiment was performed to ensure a comparability of already reported FA concentrations with those in future studies. Therefore, $35 \mathrm{hpPL}$ samples were additionally prepared for GC-FID analysis with C19:0 FAME as the IS. Twenty-seven FA concentrations, which were detected in all samples, are included into the method compare. The FA concentrations were calculated as the percentage of total lipid in the hpPL. These percentages of total lipid were directly compared by Pearson correlation. Additionally, the data were plotted within a Bland-Altman plot to compare the agreement in results between the two analytical assays. $^{26,27}$ Here, excellent correlations and low numbers of minor outliers were identified. Figure 3 shows the BlandAltman plots and correlations for the most abundant saturated, mono-unsaturated, and $\omega-3$ and $\omega-6$ polyunsaturated fatty acids.

Supplemental Table S3 shows the comparison of the absolute percentage ratio and the Bland-Altman data. Comparison between the two different analytical methods (GC-FID vs GCTQMS) showed great conformity. This is of major importance because it allows the unrestricted comparison of results from previous experiments performed with the GC-FID assay. Additionally, the absolute concentrations of low abundant and isomeric FA in hpPL are now accessible with a higher precision. Lütjohann et al. could show, in the first international ring trials for sterols and oxysterols, that the use of an inappropriate IS and the quantification of external calibration solutions are major parameters, which bias the absolute quantification. ${ }^{28,29}$ Figure 1 and Supplemental Table S2 show the absolute concentrations of FA found in hpPL of 37 samples from healthy volunteers determined by GC-TQMS. The FA C15:1n-7, C17:1n-7(t), C18:2n-9(t,t), C22:1n-9, C24:4n-6, C24:5n-6, and C24:6n-3 were not detected in every sample. Their concentration range was between 0.22 and $29.5 \mathrm{nmol} / \mathrm{mL}$. In total nine of the 58 included FA were not detected in samples of healthy volunteers.

Isotope Enrichment. By the employment of a mass selective detector, it is possible to identify changes in isotope pattern of FA in hpPL. To prove the capability to detect differences in fatty acid enrichment, we determined the ${ }^{13} \mathrm{C}$-FA 16:0 enrichment in hpPL of a human dietary experiment. Please find the results shown in Supplemental Figure S5. The utilization of a triple quadrupole mass selective detector allows also the enrichment of labeled tracers in interventional studies and enables the calculation of tracer/tracee ratio. Therefore, the here described method is a valuable tool to investigate the human fatty acid metabolism.

\section{CONCLUSIONS}

During the method development, several methodical aspects have been improved, which result in a highly robust, reproducible, and validated method for FA analysis in hpPL. The deployment of TQMS allowed the highly sensitive identification of specific fragments for the quantification and identification of FA in hpPL. This method is improved in terms of elaborate identification and precision as compared to other routinely used detection methods such as FID and MS. With the quantification of the Supelco FAME $37 \mathrm{mix}$ by GC-FID against C19:0, we could demonstrate that the introduced correction factor Rf results in correct FAME concentrations. Additionally, we can guarantee reproducible fatty acid concentrations over a long period by the possibility to quantify our stock solutions after a certain storage time. We could show that we can reliably quantify the concentrations of 58 FA over 4 orders of magnitude within one run. Results obtained from GC-FID are comparable with those achieved by the described GC-TQMS method. Finally, the method allows one to quantify fatty acid tracer/ tracee ratios within the same run as the fatty acid pattern is analyzed. The assay as described here for hpPL can easily be modified for quantification of low abundant positional and geometrical (cis/trans) isomeric FA in different human serum lipid classes. Therefore, only the extraction procedure and addition of specific labeled standards must be adapted.

\section{MATERIAL AND METHODS}

Methanol, isooctane, acetic acid, butylated hydroxytoluene, sodium chloride, pentane, diethyl ether, hexane, and boron trifluoride $\left(\mathrm{BF}_{3}\right)$ in methanol are purchased from Merck Millipore, Schiphol-Rijk, Netherlands. PC- $\mathrm{D}_{70}$ 1,2-distearoyl$\mathrm{D}_{70}$-sn-glycero-3-phosphocholine was obtained from Avanti Polar Lipids, Alabaster, Alabama, United States. A phospholipid 
mixture for HPLC from soybean containing L- $\alpha$-lysophosphatidylcholine (7.7 weight $(\mathrm{w}) \%)$, L- $\alpha$-phosphatidylcholine from soybean (38.5 w\%), L- $\alpha$-phosphatidylethanolamine (30.8 w\%), and $\mathrm{L}-\alpha$-phosphatidylinositol $(23.0 \mathrm{w} \%)$ were purchased from Sigma-Aldrich, St. Louis, Missouri, United States. A detailed list of included FA with their systematic names and a list with FAMEs and their suppliers is reported in Table 1 and Supplemental Tables S1 and S2.

Sample Preparation. Phospholipids from human EDTAplasma were extracted according to a modified method of Bligh and Dyer. ${ }^{30}$ The extraction protocol in brief: $1 \mathrm{~mL}$ of chloroform (50 mg/L butylated hydroxytoluene (BHT)), $2 \mathrm{~mL}$ of methanol (50 mg/L BHT), $900 \mu \mathrm{L}$ of aqueous 1.0\% EDTA solution, and $100 \mu \mathrm{L}$ of $\mathrm{D}_{70}-\mathrm{C} 18: 0 \mathrm{PC}(2 \mathrm{mM} / \mathrm{L})$ were added to $100 \mu \mathrm{L}$ of plasma sample and vortexed for $10 \mathrm{~min}$. Additional $1 \mathrm{~mL}$ of $1.0 \%$ EDTA solution and $1 \mathrm{~mL}$ chloroform were added and vortexed for further $2 \mathrm{~min}$. Consecutively, the sample was centrifuged for 10 min with $10.000 \mathrm{rpm}$ by $4{ }^{\circ} \mathrm{C}$ and the lower organic phase was collected. Additional $2 \mathrm{~mL}$ of chloroform was added, vortexed for $10 \mathrm{~min}$, and centrifuged as described above. The two lower organic phases were combined and dried under nitrogen at 30 ${ }^{\circ} \mathrm{C}$. hpPL were purified from the lipid extract with aminopropyl solid phase extraction by a modified protocol of Kaluzny et al.: ${ }^{31}$ (in brief) Aminopropyl columns (500 mg) were placed in the Vac Elut apparatus and washed twice with $2 \mathrm{~mL}$ portions of methanol $(50 \mathrm{mg} / \mathrm{L} \mathrm{BHT})$ and twice with $2 \mathrm{~mL}$ portions of $n$ hexane $(50 \mathrm{mg} / \mathrm{L} \mathrm{BHT})$ under reduced pressure and twice with $2 \mathrm{~mL}$ of $n$-hexane under normal pressure. The sample was reconstituted twice in $250 \mu \mathrm{L}$ of chloroform and applied to the column under reduced pressure and washed with $2 \times 2.0 \mathrm{~mL}$ of $2 \%$ acetic acid in diethyl ether. PL were eluted from the solid phase by $2 \times 2.0 \mathrm{~mL}$ of methanol ( $50 \mathrm{mg} / \mathrm{L} \mathrm{BHT})$ under reduced pressure. This solution was evaporated under nitrogen at $37^{\circ} \mathrm{C}$ and kept at $-20{ }^{\circ} \mathrm{C}$ until methylation. The PL were hydrolyzed, and the resulting FA were methylated to their corresponding methyl esters by reaction with BF3 $(100 \mathrm{~g} / \mathrm{L})$ in methanol at 100 ${ }^{\circ} \mathrm{C}$ for $1 \mathrm{~h} .{ }^{24,32}$ The sample was reconstituted in $100 \mu \mathrm{L}$ of isooctane for analysis by GC-TQMS and $20 \mu \mathrm{L}$ for GC-FID, respectively.

Gas Chromatographic-Triple Quadrupole Mass Spectrometric Quantifications of Fatty Acid Methyl Esters. GC-TQMS analyses of the FAMEs were performed on an Agilent 7000 TQMS connected to an Agilent 7890A gas chromatograph equipped with an autosampler. FAMEs were separated by a highly polar CP-Sil88 column (100 m in length, $0.25 \mathrm{~mm}$ in diameter, and $<0.192 \mu \mathrm{m}$ film thickness, Agilent $\mathrm{J} \& W$ ) coated with a stabilized cyanopropyl phase for the separation of positional and geometric isomers. The injection volume was $1 \mu \mathrm{L}$, and the GC inlet was operated in pulsed splitless mode by $300{ }^{\circ} \mathrm{C}$, with 40 psi pulse until $0.5 \mathrm{~min}$. The liner was packed with glass wool (Agilent). The temperature program was as follows: the initial oven temperature of $85{ }^{\circ} \mathrm{C}$ was held for $2.0 \mathrm{~min}$, then increased with $100^{\circ} \mathrm{C} / \mathrm{min}$ to $164^{\circ} \mathrm{C}$ held until $21.0 \mathrm{~min}$, with $2{ }^{\circ} \mathrm{C} / \mathrm{min}$ to $190{ }^{\circ} \mathrm{C}$ held for $6.0 \mathrm{~min}$, and reached finally a temperature of $230^{\circ} \mathrm{C}$ with $15^{\circ} \mathrm{C} / \mathrm{min}$ and held for $40 \mathrm{~min}$. Helium was used as a carrier gas with a constant linear velocity of $53.67 \mathrm{~mL} / \mathrm{min}$. Aux and transfer line temperatures were set to $300{ }^{\circ} \mathrm{C}$. The temperature of the MS ion source and both quadrupoles were maintained at 230 and $150{ }^{\circ} \mathrm{C}$, respectively. The MS was operated at $70 \mathrm{eV}$ and in multiple reaction mode (MRM) mode. The FAME profiles were corrected for blank runs. As the IS for absolute quantification, $\mathrm{D}_{35}$-C18:0 was used.
Gas Chromatographic-Flame Ionization Quantifications of Fatty Acid Methyl Ester. GC-FID analysis of the FAMEs were performed as previously described by Wensing et al. using an GC2010 plus (Shimadzu, Den Bosch Netherlands) GC, equipped with a $50 \mathrm{~m}$ CP-Sil88 polar capillary column with a $0.25 \mathrm{~mm}$ i.d. and film thickness of $<0.192 \mu \mathrm{m}$ (Agilent J\&W) using a split ratio of $1: 20 .{ }^{24}$ Helium was used as a carrier gas (130 $\mathrm{kPa})$, and the injection and detection temperatures were both set to $300{ }^{\circ} \mathrm{C}$. The starting temperature of the column was 160 ${ }^{\circ} \mathrm{C}$. At $10 \mathrm{~min}$ after injection, the temperature was increased to $190{ }^{\circ} \mathrm{C}$ at a rate of $5{ }^{\circ} \mathrm{C} / \mathrm{min}$; after $15 \mathrm{~min}$ at $190{ }^{\circ} \mathrm{C}$, the temperature was increased to $230^{\circ} \mathrm{C}$ at a rate of $5{ }^{\circ} \mathrm{C} / \mathrm{min}$. The final temperature of $230{ }^{\circ} \mathrm{C}$ was maintained for $7 \mathrm{~min}$. The FAME profiles were corrected for blank runs. The FAME C19:0 was used as the IS.

Quantification of Customized External Calibration Solution. For absolute quantification of FA pattern in hpPL an external calibration solution was custom prepared. A purity check in the commercially available FAME standards stocks by analysis with GC-FID or GC-TQMS full scan revealed no major disturbing cross-contamination with FAMEs. The concentrations of FAME standards were determined in the three different calibration stock solutions composed for baseline separation by the GC-FID method and quantified against $50 \mu \mathrm{L}$ of C19:0 solution $(2 \mathrm{mg} / \mathrm{mL})$ used as the IS. For GC-TQMS analysis, these three stock solutions were combined and diluted and used for external calibration. Due to the differences in the number of carbon atoms and hydrogen saturation, it was necessary to introduce a correction factor (Rf) for the quantification of the 58 FAME standards. This factor $\mathrm{Rf}$ was used to include the individual structural differences of carbon number and saturation of the FAMEs and the IS for quantification C19:0. Please find equations for calculation of the correction factor and the individual $\mathrm{Rf}$ values in Supplemental Equation $\mathrm{S} 1$ and Supplemental Table S1.

Method Validation. The method validation was realized according to the FDA and EMA guidelines for the validation of bioanalytical methods. ${ }^{17,18}$ Both guidelines refer to typical validation characteristics such as selectivity, linearity, limits of detection (LOD) and limits of quantification (LOQ), precision (intraday variability, between day variability), and recovery. Detailed validation protocol is shown in Supplemental Section S3.

Sample Collection for Method Comparison. Fatty acid compositions of hpPL were analyzed in 37 samples from a population of healthy individuals obtained at baseline of a placebo-controlled intervention study by Pieters et al.. ${ }^{33}$ Sample collection for isotope enrichment analysis: Samples for analyzing ${ }^{13} \mathrm{C}-16: 0$ enrichment and calculating ${ }^{13} \mathrm{C}$-tracer/tracee ratios were obtained from a study earlier performed by Lindeboom et al.. ${ }^{34}$ A detailed list of the inclusion parameters and volunteer recruitment is shown in Supplemental Section S4. All procedures involving human subjects were approved by the Medical Ethical Committee of Maastricht University. Written informed consent was obtained from all subjects before the start of the study.

Statistical Analyses. Data for linearity evaluation were tested for normal and Gaussian distribution. $P$-values of $<0.05$ were considered statistically significant. All statistical tests were performed with SPSS 21 (Chicago, Illinois) and Excel 10. 


\section{ASSOCIATED CONTENT}

\section{s) Supporting Information}

The Supporting Information is available free of charge at https://pubs.acs.org/doi/10.1021/acsomega.0c03874.

Supplementary methods, results, and appendices (PDF)

\section{AUTHOR INFORMATION}

\section{Corresponding Authors}

Hans-F. Schött - Singapore Lipidomics Incubator (SLING), Department of Biochemistry, Yong Loo Lin School of Medicine, National University of Singapore, 117597, Singapore; Department of Nutrition and Movement Sciences, Maastricht University, 6200 MD Maastricht, The Netherlands; ○ orcid.org/0000-0003-3907-3816; Email: bchshf@ nus.edu.sg

Jogchum Plat - Department of Nutrition and Movement Sciences, Maastricht University, 6200 MD Maastricht, The Netherlands; Email: j.plat@maastrichtuniversity.nl

\section{Authors}

Maurice C. J. M. Konings - Department of Nutrition and Movement Sciences, Maastricht University, 6200 MD Maastricht, The Netherlands

Vera B. Schrauwen-Hinderling - Department of Nutrition and Movement Sciences, Maastricht University, 6200 MD Maastricht, The Netherlands

Ronald P. Mensink - Department of Nutrition and Movement Sciences, Maastricht University, 6200 MD Maastricht, The Netherlands

Complete contact information is available at:

https://pubs.acs.org/10.1021/acsomega.0c03874

\section{Author Contributions}

${ }^{\S}$ H.S. and M.K. contributed equally.

\section{Author Contributions}

H.S. and M.K. conducted the analysis; H.S., M.K., and J.P. analyzed the data and wrote the paper; J.P. had primary responsibility for the final content. V.S.H. was responsible for the isotope enrichment samples. R.M. was responsible for the comparison samples. All authors read and approved the final manuscript.

\section{Notes}

The authors declare no competing financial interest.

\section{ACKNOWLEDGMENTS}

Fatty acid study: R.P.M. was responsible for the samples from the study used for method comparison. The authors thank all volunteers for participating in the study and Daisy Luiten for technical support. This work was partially funded by Unilever Research and Development Vlaardingen, The Netherlands. Isotope study: V.B.S.H. was responsible for the samples from this study used here. The authors thank all volunteers for participating in the study. This study was funded by TI Food and Nutrition, a public-private partnership on precompetitive research in food and nutrition. In both studies, the public partners were responsible for the study design, data collection and analysis, decision to publish, and preparation of the manuscript.

\section{TABLE OF CONTENT}

FA

fatty acids
GC gas chromatography

TQMS triple quadrupole mass selective detection

GC-TQMS gas chromatography-triple quadrupole mass selective detection

hpPL human plasma phospholipids

FID flame ionization detection

GC-FID gas chromatography-flame ionization detection

PL phospholipids

IS internal standard

EI electron impact

LOD limits of detection

LOQ limits of quantification

MS single quadrupole mass selective detector

MRM multiple reaction mode

\section{REFERENCES}

(1) Calder, P. C. Functional Roles of Fatty Acids and Their Effects on Human Health. J. Parenter. Enteral Nutr. 2015, 39, 18S-32S.

(2) Lecerf, J.-M. Fatty acids and cardiovascular disease. Nutr. Rev. 2009, 67, 273-283.

(3) Reiffel, J. A.; McDonald, A. Antiarrhythmic effects of omega-3 fatty acids. Am. J. Cardiol. 2006, 98, 50-60.

(4) Sanders, T. A. B. Omega-6 Fatty Acids and Cardiovascular Disease. Circulation 2019, 139, 2437-2439.

(5) Dennis, E. A.; Norris, P. C. Eicosanoid Storm in Infection and Inflammation. Nat. Rev. Immunol. 2015, 15, 511-523.

(6) Lutz, B.; Marsicano, G.; Maldonado, R.; Hillard, C. J. The endocannabinoid system in guarding against fear, anxiety and stress. Nat. Rev. Neurosci. 2015, 16, 705-718.

(7) Sharkey, K. A.; Wiley, J. W. The Role of the Endocannabinoid System in the Brain-Gut Axis. Gastroenterology 2016, 151, 252-266.

(8) Micha, R.; Mozaffarian, D. Trans fatty acids: effects on metabolic syndrome, heart disease and diabetes. Nat. Rev. Endocrinol. 2009, 5, 335-344.

(9) Mozaffarian, D.; Aro, A.; Willett, W. C. Health effects of trans-fatty acids: experimental and observational evidence. Eur. J. Clin. Nutr. 2009, 63, S5-S21.

(10) Thijssen, M. A.; Mensink, R. P. Fatty acids and atherosclerotic risk. Handb. Exp. Pharmacol. 2005, 170, 165-194.

(11) Uauy, R.; Aro, A.; Clarke, R.; Ghafoorunissa; L’Abbé, M. R.; Mozaffarian, D.; Skeaff, C. M.; Stender, S.; Tavella, M. WHO Scientific Update on trans fatty acids: summary and conclusions. Eur. J. Clin. Nutr. 2009, 63, S68-S75.

(12) Skeaff, C. M.; Miller, J. Dietary fat and coronary heart disease: summary of evidence from prospective cohort and randomised controlled trials. Ann. Nutr. Metab. 2009, 55, 173-201.

(13) Kish-Trier, E.; Schwarz, E. L.; Pasquali, M.; Yuzyuk, T. Quantitation of total fatty acids in plasma and serum by GC-NCIMS. Clin. Mass Spectrom. 2016, 2, 11-17.

(14) Xu, Y.-J.; Zhang, J. Determination of fatty acid methyl esters by GC-triple quadrupole MS using electron and chemical ionization. Bioanalysis 2013, 5, 1527-1543.

(15) Khaw, K.-T.; Friesen, M. D.; Riboli, E.; Luben, R.; Wareham, N. Plasma phospholipid fatty acid concentration and incident coronary heart disease in men and women: the EPIC-Norfolk prospective study. PLoS Med. 2012, 9, No. e1001255.

(16) de Saint Laumer, J.-Y.; Leocata, S.; Tissot, E.; Baroux, L.; Kampf, D. M.; Merle, P.; Boschung, A.; Seyfried, M.; Chaintreau, A. Prediction of response factors for gas chromatography with flame ionization detection: Algorithm improvement, extension to silylated compounds, and application to the quantification of metabolites. J. Sep. Sci. 2015, 38, 3209-3217.

(17) U.S. Department of Health and Human Services Food and Drug Administration. Guidance for Industry Bioanalytical Method Validation DRAFT GUIDANCE; US Department of Health and Human Services: 2013. 
(18) European Medicines Agency. Guideline on bioanalytical method validation; 2011.

(19) Ecker, J.; Scherer, M.; Schmitz, G.; Liebisch, G. A rapid GC-MS method for quantification of positional and geometric isomers of fatty acid methyl esters. J. Chromatogr. B: Anal. Technol. Biomed. LLife Sci. 2012, 897, 98-104.

(20) Mossoba, M. M.; Krammer, J. K. G.; Delmonte, P.; Yurawecz, M.; Rader, J.. AOAC Official Method 996.06 Fat (Total, Saturated, and Unsaturated) in Foods:Hydrolytic Extraction Gas Chromatographic Method First Action 1996, Revised 2001; AOCS Press: 2003.

(21) Kopf, T.; Schmitz, G. Analysis of non-esterified fatty acids in human samples by solid-phase-extraction and gas chromatography/ mass spectrometry. J. Chromatogr. B: Anal. Technol. Biomed. Life Sci. 2013, 938, 22-26.

(22) Ren, J.; Mozurkewich, E. L.; Sen, A.; Vahratian, A. M.; Ferreri, T. G.; Morse, A. N.; Djuric, Z. Total Serum Fatty Acid Analysis by GCMS: Assay Validation and Serum Sample Stability. Curr. Pharm. Anal. 2013, 9, 331-339.

(23) Thurnhofer, S.; Vetter, W. A gas chromatography/electron ionization-mass spectrometry-selected ion monitoring method for determining the fatty acid pattern in food after formation of fatty acid methyl esters. J. Agric. Food Chem. 2005, 53, 8896-8903.

(24) Wensing, A. G. C. L.; Mensink, R. P.; Hornstra, G. Effects of dietary n-3 polyunsaturated fatty acids from plant and marine origin on platelet aggregation in healthy elderly subjects. J. Geophys. Res. Oceans 1999, 82, 183-191.

(25) Pacchiarotta, T.; Nevedomskaya, E.; Carrasco-Pancorbo, A.; Deelder, A. M.; Mayboroda, O. A. Evaluation of GC-APCI/MS and GC-FID as a complementary platform. J. Biomol. Tech. 2010, 21, 205213.

(26) Bland, J. M.; Altman, D. G. Statistical methods for assessing agreement between two methods of clinical measurement. Lancet 1986, $1,307-310$.

(27) Bland, J. M.; Altman, D. G. Measuring agreement in method comparison studies. Stat. Methods Med. Res. 1999, 8, 135-160.

(28) Lütjohann, D.; Björkhem, I.; Friedrichs, S.; Kerksiek, A.; Geilenkeuser, W.-J.; Lövgren-Sandblom, A.; Ansorena, D.; Astiasarán, I.; Baila-Rueda, L.; Barriuso, B.; Bretillon, L.; Browne, R. W.; Caccia, C.; Cenarro, A.; Crick, P. J.; Fauler, G.; García-Llatas, G.; Griffiths, W. J.; Iuliano, L.; Lagarda, M. J.; Leoni, V.; Lottenberg, A. M.; Matysik, S.; McDonald, J.; Rideout, T. C.; Schmitz, G.; Nunes, V. S.; Wang, Y.; Zerbinati, C.; Diczfalusy, U.; Schött, H.-F. International descriptive and interventional survey for oxycholesterol determination by gas- and liquid-chromatographic methods. Biochimie 2018, 153, 26-32.

(29) Lütjohann, D.; Björkhem, I.; Friedrichs, S.; Kerksiek, A.; Lövgren-Sandblom, A.; Geilenkeuser, W.-J.; Ahrends, R.; Andrade, I.; Ansorena, D.; Astiasarán, I.; Baila-Rueda, L.; Barriuso, B.; Becker, S.; Bretillon, L.; Browne, R. W.; Caccia, C.; Ceglarek, U.; Cenarro, A.; Crick, P. J.; Fauler, G.; Garcia-Llatas, G.; Gray, R.; Griffiths, W. J.; Gylling, H.; Harding, S.; Helmschrodt, C.; Iuliano, L.; Janssen, H.-G.; Jones, P.; Kaipiainen, L.; Kannenberg, F.; Lagarda, M. J.; Leoni, V.; Lottenberg, A. M.; Mackay, D. S.; Matysik, S.; McDonald, J.; Menendez-Carreño, M.; Myrie, S. B.; Sutti Nunes, V.; Ostlund, R. E.; Polisecki, E.; Ramos, F.; Rideout, T. C.; Schaefer, E. J.; Schmitz, G.; Wang, Y.; Zerbinati, C.; Diczfalusy, U.; Schött, H.-F. First international descriptive and interventional survey for cholesterol and noncholesterol sterol determination by gas- and liquid-chromatography Urgent need for harmonisation of analytical methods. J. Steroid Biochem. Mol. Biol. 2019, 190, 115-125.

(30) Bligh, E. G.; Dyer, W. J. A rapid method of total lipid extraction and purification. Can. J. Biochem. Physiol. 1959, 37, 911-917.

(31) Kaluzny, M. A.; Duncan, L. A.; Merritt, M. V.; Epps, D. E. Rapid separation of lipid classes in high yield and purity using bonded phase columns. J. Lipid Res. 1985, 26, 135-140.

(32) Morrison, W. R.; Smith, L. M. Preparation of Fatty Acid Methyl Esters and Dimethylacetals from Lipids with Boron Fluoride-Methanol. J. Lipid Res. 1964, 5, 600-608.

(33) Pieters, D. J.; Zock, P. L.; Fuchs, D.; Mensink, R. P. Effect of $\alpha$ linolenic acid on 24-h ambulatory blood pressure in untreated high- normal and stage I hypertensive subjects. J. Geophys. Res. Oceans 2019, 121, 155-163.

(34) Lindeboom, L.; de Graaf, R. A.; Nabuurs, C. I.; van Ewijk, P. A.; Hesselink, M. K. C.; Wildberger, J. E.; Schrauwen, P.; SchrauwenHinderling, V. B. Quantum coherence spectroscopy to measure dietary fat retention in the liver. JCI insight 2016, 1, No. e84671. 